\title{
The chiral phase transition and the role of vacuum fluctuations
}

\author{
Jens O. Andersen $⿴$ Rashid Khan $才$ and Lars T. Kyllingstad \\ Department of Physics, Norwegian Institute of Science and Technology, N-7491 Trondheim, Norway
}

(Dated: October 22, 2018)

\begin{abstract}
We apply optimized perturbation theory to the quark-meson model at finite temperature $T$ and quark chemical potential $\mu$. The effective potential is calculated to one loop both in the chiral limit and at the physical point and used to study the chiral dynamics of two-flavor QCD. The critical temperature and the order of the phase transition depends heavily on whether or not one includes the bosonic and fermionic vacuum fluctuations in the effective potential. A full one-loop calculation in the chiral limit predicts a first-order transition for all values of $\mu$. At the physical point, one finds a crossover in the whole $\mu-T$ plane.
\end{abstract}

PACS numbers: 21.65Qr;25.75Nq

\section{INTRODUCTION}

If we consider QCD in the chiral limit, the QCD Lagrangian has an extended symmetry. For $N_{f}$ quark flavors, the Lagrangian is invariant under $S U\left(N_{f}\right)_{L} \times S U\left(N_{f}\right)_{R}$ transformations. In the vacuum, this symmetry is spontaneously broken down to $S U\left(N_{f}\right)_{V}$ by the formation of a chiral condensate. According to Goldstone's theorem, this gives rise to $N_{f}^{2}-1$ massless modes, the Nambu-Goldstone bosons.

In the real world, quarks are not massless. If we add a mass term for the quarks to the QCD Lagrangian, chiral symmetry is explicitly broken, which means that the Goldstone bosons become massive as well. Nevertheless, the $u$ and $d$ quarks are light enough that we have an approximate chiral symmetry in Nature, and for simplicity, we therefore often consider them to be massless. QCD with two flavors of massless quarks is a commonly used approximation, the validity of which seems to be supported by the observed lightness of the pions. However, there are more than two quark flavors in the real world. In particular, the strange quark mass is on the order of $100 \mathrm{MeV}$, and so we expect it to have an impact on physics at this energy scale.

If one heats strongly interacting matter, it is expected that there will be a transition to a phase where chiral symmetry is restored and the quarks within the hadrons are liberated. The location and nature of the transition from the phase with broken chiral symmetry to the chirally symmetric phase is a topic of active research. The order of the phase transition depends on the number of quark flavors and their masses. For example, for two quark flavors and vanishing baryon number chemical potential $\mu=0$, we expect, a second-order phase transition in the chiral limit if the $U(1)_{A}$ is explicitly broken, otherwise it is driven first order. In the case of finite quark masses it is a crossover. For three massless quarks, $N_{f}=3$, the transition is always first order. These results follow from universality-class arguments and the (non)existence of stable fixed points [1, 2]. At nonzero baryon chemical potential and in particular at $T=0$, the order of the chiral transition is not obvious from universality arguments [3]. However, most model calculations predict a first-order transition at $T=0$ [2, 4]. If this is the case, there is a line of first order chiral transitions in the $\mu-T$ plane that ends in a second-order transition.

Currently, the only way to obtain quantitative information about the chiral phase transition from first principles is to use lattice Monte Carlo simulations. Results from lattice QCD indicate that the transition takes place at $T_{c} \sim 150 \mathrm{MeV}$ depending on the number and masses of the quarks [5, [6].

Unfortunately, lattice QCD is severely limited by the sign problem. When the baryon number chemical potential is nonzero, the fermion determinant, which is used as the statistical weight in the Monte Carlo importance sampling, becomes complex. There are several methods to sidestep the sign problem for small $\mu$. Examples include reweighting techniques, Taylor expansions in $\mu$ around $\mu=0$, and the use of imaginary chemical potentials. For a review, see

\footnotetext{
*Electronic address: andersen@tf.phys.no

${ }^{\dagger}$ Electronic address: rashid.khan@ntnu.no

${ }^{\ddagger}$ Electronic address: Lars.kyllingstad@ntnu.no
} 
Ref. [7]. However, these methods only work for $\mu \lesssim T$, and a general solution to the sign problem for all $\mu$ has yet to be found.

A complementary approach to the investigation of the chiral phase transition, which works even at nonzero baryon number chemical potential, is offered by effective models - simplified models that share QCD's chiral symmetry breaking pattern. Examples include linear sigma models [8 12], NJL-type models [13 17], Polyakov-NJL models 18] the quark-meson (QM) effective model ${ }^{1}$ [13, 19 28], (see also Ref. 29]), and the Polyakov-QM model [16, 30 37]. In this paper we will employ the QM model.

By definition, when using effective models we neglect certain aspects of the full theory. In $\chi \mathrm{PT}$ and sigma models, for instance, we only have mesonic degrees of freedom. For this reason, there is no way to include a nonzero baryon number chemical potential in these models.

In NJL-type models we have quark degrees of freedom, however, the color symmetry is global, which means that there are no gluon degrees of freedom. One-gluon exchange has been replaced by local four-fermion interactions between the quarks. The NJL model is not confining and the quarks are not asymptotically free [4]. Unlike the scalar models above, it allows the inclusion of separate chemical potentials for different quark flavors (and colors), and through the use of NJL models a rich structure of color superconducting phases at $\mu \neq 0$ and low temperature has emerged [38].

While the effective models themselves are already simplifications of a more complicated theory, one often makes additional simplifications within the models. For qualitative considerations, for instance, one may decide to omit contributions that do not seem immediately important for the problem at hand. As an example, in the QM model, chiral symmetry breaking takes place in the meson sector, and as a consequence, the vacuum contribution to the free energy from the fermions is sometimes omitted. In the NJL model, on the other hand, this term is responsible for the chiral symmetry breaking, and so it cannot be neglected.

When making such simplifications, however, it is important to know exactly what one is discarding, in order not to "throw the baby out with the bath water". To wit, for the example just given, it was recently shown that neglecting the fermion vacuum contribution to the QM free energy changes the order of the phase transition [26]. In the present paper we will study the role of the vacuum fluctuations in detail and generalize to $\mu \neq 0$.

The quark-meson effective model contains, as the name implies, both quark and meson degrees of freedom. The mesons are described as in the linear sigma model, by an $O(N)$-symmetric scalar field theory with a quartic selfinteraction. The meson sector couples to the quark sector through Yukawa-type couplings.

We shall investigate the thermodynamics of the QM model using optimized perturbation theory (OPT), which was introduced in 1998 by Chiku and Hatsuda [8, 10]. In this framework, a local quadratic term proportional to an arbitrary parameter $\chi$ is added to and subtracted from the Lagrangian [8, 10, 40 48] In the perturbative expansion, the added mass term is included in the free part of the Lagrangian and the subtracted term is treated as an interaction term. This constitutes a reorganisation of the perturbative series in which higher-order terms of naïve perturbation theory are resummed to all orders.

We have already mentioned that the pions are the Nambu-Goldstone bosons associated with the spontaneous breaking of chiral symmetry. As such, the Nambu-Goldstone theorem plays a significant role in the study of the chiral phase transition. Importantly, OPT ensures that this theorem is satisified order-by-order of the expansion [10].

The paper is organized as follows: In Sec. [I] we present the quark-meson effective model and the OPT framework; in Sec. III], we calculate the free energy to one loop; in Sec.[V] we present our results and in Sec. $\nabla$, we make a few concluding remarks. Finally, expressions for various sum-integrals are provided in an appendix.

\section{QUARK-MESON MODEL}

We consider the quark-meson effective model with $N$ real scalar fields $\left(\phi_{1}, \phi_{2}, \ldots, \phi_{N}\right)$ and $N_{f}$ massless quark flavors $(\psi)$ with $N_{c}$ colors. The bare Euclidean Lagrangian for the model can be written in the form

$$
\mathcal{L}_{B}=\mathcal{L}+\Delta \mathcal{L}
$$

\footnotetext{
1 The QM model is also known as the linear sigma model coupled to quarks (LSMq).
} 
where $\mathcal{L}$ is the renormalized Lagrangian and $\Delta \mathcal{L}$ contains the counterterms. For clarity, we further write $\mathcal{L}$ as

$$
\mathcal{L}=\mathcal{L}_{\mathrm{LSM}}+\mathcal{L}_{\mathrm{q}}+\mathcal{L}_{\text {Yukawa }}
$$

The first term in this expression, $\mathcal{L}_{\mathrm{LSM}}$, is the Lagrangian of the linear sigma model:

$$
\mathcal{L}_{\mathrm{LSM}}=\frac{1}{2}\left(\partial_{\mu} \phi_{i}\right)\left(\partial_{\mu} \phi_{i}\right)+\frac{1}{2} m_{0}^{2} \phi_{i} \phi_{i}+\frac{\lambda}{4 !}\left(\phi_{i} \phi_{i}\right)^{2}-h \phi_{1} .
$$

In the case of vanishing $h$, this Lagrangian has an $O(N)$ symmetry which, assuming $m_{0}^{2}<0$, is spontaneously broken down to $O(N-1)$. When $h \neq 0$ the symmetry is explicitly broken, mimicking the explicit breaking of chiral symmetry in the QCD Lagrangian by nonzero quark masses.

Next, we have $\mathcal{L}_{\mathrm{q}}$, describing the massless quarks:

$$
\mathcal{L}_{\mathrm{q}}=\bar{\psi}\left(\gamma_{\mu} \partial_{\mu}-\mu \gamma_{4}\right) \psi .
$$

Here, $\mu$ is the quark number chemical potential, which we assume to be equal for all quark flavors, and which is related to the baryon number chemical potential by $\mu=\mu_{B} / 3 . \mathcal{L}_{\mathrm{q}}$ has a $U(1)_{B}$ symmetry related to the conservation of baryon number, as well as an $S U\left(N_{f}\right)_{L} \times S U\left(N_{f}\right)_{R}$ chiral symmetry.

In Eq. (4) and throughout the remainder of the paper, we take $\gamma_{\mu}$ to denote Euclidean gamma matrices. These are related to the familiar gamma matrices in Minkowski space by $\gamma_{i} \equiv i \gamma_{M}^{i}$ and $\gamma_{4} \equiv \gamma_{M}^{0}$. They have the following properties: $\left\{\gamma_{\mu}, \gamma_{\nu}\right\}=2 \delta_{\mu \nu} \mathbf{1}$ and $\operatorname{tr}\left[\gamma_{\mu} \gamma_{\nu}\right]=4 \delta_{\mu \nu}$.

$\mathcal{L}_{\text {Yukawa }}$ describes the Yukawa couplings between the scalars and the quarks, and we write it compactly in the form

$$
\mathcal{L}_{\text {Yukawa }}=g \bar{\psi} \Gamma_{i} \psi \phi_{i}
$$

Here, we have defined

$$
\Gamma \equiv\left(1,-i \gamma_{5} \tau\right)
$$

where $\tau=\left(\tau_{1}, \tau_{2}, \tau_{3}\right)$ are the Pauli matrices. This only makes sense when $N=4$, which is the value we will use in the end. The fifth Euclidean gamma matrix is defined as $\gamma_{5} \equiv \gamma_{1} \gamma_{2} \gamma_{3} \gamma_{4}=-\gamma_{M}^{5}$. Finally, the counterterm Lagrangian $\Delta \mathcal{L}$ is written as

$$
\Delta \mathcal{L}=\frac{1}{2} A\left(\partial_{\mu} \phi_{i}\right)\left(\partial_{\mu} \phi_{i}\right)+\frac{1}{2} B m_{0}^{2} \phi_{i} \phi_{i}+\frac{C \lambda}{4 !}\left(\phi_{i} \phi_{i}\right)^{2}-D m_{0}^{4}+F \bar{\psi} \gamma_{\mu} \partial_{\mu} \psi+G g \bar{\psi} \Gamma_{i} \psi \phi_{i},
$$

where $A, B, C, D, F$, and $G$ are the renormalisation constants for the scalar field, the scalar mass, the four-scalar coupling, the vacuum energy, the fermion field, and the Yukawa coupling, respectively. In the present calculation, we need to know $B, C$, and $D$ to leading order in the couplings.

\section{A. Broken symmetry}

The group $O(4)$ is homomorphic to $S U(2) \times S U(2)$. The consequence of this is that if we take $N=4$ and $N_{f}=2$, an $O(4)$ rotation among the scalar fields can always be countered by an $S U(2) \times S U(2)$ transformation on the fermions, keeping the entire Lagrangian invariant.

Furthermore, $O(3)$, the symmetry group of $\mathcal{L}_{\mathrm{LSM}}$ in the broken phase, is homomorphic to $S U(2)$, which is the symmetry group of $\mathcal{L}_{\mathrm{q}}$ after chiral symmetry has been broken by, say, the addition of a nonzero mass term. Indeed, we shall see that spontaneous symmetry breaking in the mesonic sector in fact induces a dynamic mass term for the fermions.

In the phase with broken chiral symmetry, the scalar field $\phi$ takes on a nonzero vacuum expectation value, and provided $h>0$, the vacuum state will always point in the $\phi_{1}$ direction. When $h=0, O(N)$ symmetry allows us to choose any direction for the vacuum state, and for simplicity we choose the same direction in this case. We therefore write

$$
\phi=(\sigma+v, \pi),
$$

where $\sigma$ and $\pi$ are fluctuations representing the sigma meson and the pions, while $v$ is the vacuum expectation value of $\phi_{1}$. 


\section{B. Optimized perturbation theory}

In optimized perturbation theory (OPT), we add and subtract quadratic terms in the Lagrangian:

$$
\mathcal{L} \rightarrow \mathcal{L}+\frac{1}{2} \chi \sigma^{2}+\frac{1}{2} \chi \pi_{i} \pi_{i}-\frac{1}{2} \chi \sigma^{2}-\frac{1}{2} \chi \pi_{i} \pi_{i}
$$

The first two terms are treated as part of the free Lagrangian and make this explicit by absorbing them into a new mass parameter $m$, defined by

$$
m^{2} \equiv m_{0}^{2}+\chi
$$

The subtracted terms are treated as two-particle interactions. Optimized perturbation theory is then defined by the counting rule that an insertion of $\chi$ counts as a factor of $\lambda$ or $g^{2}$ in loop diagrams.

Note that we must take care to adjust the counterterms as well. For instance,

$$
\Delta \mathcal{L}_{0}=\frac{1}{2} B\left(m^{2}-\chi\right) v^{2}+\frac{C \lambda}{4 !} v^{4}-D\left(m^{2}-\chi\right)^{2} .
$$

The optimization parameter $\chi$ is in principle completely arbitrary, and if we could carry out calculations to all orders, the results would be independent of $\chi$. In order to complete a calculation, we therefore need a prescription for $\chi$. There are infinitely many possible choices, but we limit ourselves to mentioning the following two prescriptions:

- The principle of minimal sensitivity (PMS) says that one should try to minimize the dependence of the result on the parameter $\chi$. Thus, a PMS condition on some observable calculated to $\ell$ loops, $\mathcal{O}_{\ell}$, would then be expressed as

$$
\frac{d \mathcal{O}_{\ell}}{d m}=0
$$

- A fastest apparent convergence (FAC) condition means that the perturbative corrections in $\mathcal{O}_{\ell}$ should be as small as possible. Sometimes it is even possible to enforce the strict condition that

$$
\mathcal{O}_{\ell}-\mathcal{O}_{\ell-n}=0
$$

for some $n$ satisfying $1 \leq n \leq \ell$.

\section{FREE ENERGY}

We now calculate the free energy $\mathcal{F}$ to one loop. In the following, we employ the notation

$$
\begin{aligned}
\mathcal{F}_{P} & \equiv\left(\frac{e^{\gamma} \Lambda^{2}}{4 \pi}\right)^{\epsilon} T \sum_{P_{0}=2 \pi n T} \int_{p} \frac{d^{d} p}{(2 \pi)^{d}}, \\
\mathcal{Y}_{\{P\}} & \equiv\left(\frac{e^{\gamma} \Lambda^{2}}{4 \pi}\right)^{\epsilon} T \sum_{P_{0}=(2 n+1) \pi T+i \mu} \int_{p} \frac{d^{d} p}{(2 \pi)^{d}},
\end{aligned}
$$

for the dimensionally regularized sum-integral, where $d=3-2 \epsilon$ is the dimension of space and $\Lambda$ is an arbitrary momentum scale. The factor $e^{\gamma} / 4 \pi$ is chosen so that, after subtraction of the poles in $\epsilon, \Lambda$ coincides with the $\overline{\text { MS }}$ renormalization scale.

To make the various contributions to the prefactors of the diagrams as explicit as possible, we take the number of scalar fields to be $N$ (i.e. $(N-1)$ pion fields) and the number of quarks to be $N_{f}$.

At tree-level, the free energy is given by

$$
\mathcal{F}_{0}=\frac{1}{2} m^{2} v^{2}+\frac{\lambda}{4 !} v^{4}-h v .
$$


To one-loop order, we obtain

$$
\mathcal{F}_{1}=\mathcal{F}_{1 \mathrm{a}}+\mathcal{F}_{1 \mathrm{~b}}+\mathcal{F}_{1 \mathrm{c}}-\frac{1}{2} \chi v^{2}+\Delta \mathcal{F}_{1}
$$

where

$$
\begin{aligned}
& \mathcal{F}_{1 \mathrm{a}}=\frac{1}{2} \mathcal{F}_{P} \log \left(P^{2}+m_{\sigma}^{2}\right), \\
& \mathcal{F}_{1 b}=\frac{1}{2}(N-1) \mathcal{f}_{P} \log \left(P^{2}+m_{\pi}^{2}\right), \\
& \mathcal{F}_{1 c}=-N_{c} N_{f} \mathcal{f}_{\{P\}} \operatorname{tr} \log \left(i \gamma_{\mu} P_{\mu}+m_{q}-\mu \gamma_{4}\right),
\end{aligned}
$$

and $\Delta \mathcal{F}_{1}$ is the one-loop counterterm:

$$
\Delta \mathcal{F}_{1}=\frac{1}{2} B_{1} m^{2} v^{2}+\frac{C_{1} \lambda}{4 !} v^{4}-D_{1} m^{4} .
$$

The masses are

$$
\begin{aligned}
& m_{\sigma}^{2}=m^{2}+\frac{\lambda}{2} v^{2}, \\
& m_{\pi}^{2}=m^{2}+\frac{\lambda}{6} v^{2}, \\
& m_{q}^{2}=g^{2} v^{2} .
\end{aligned}
$$

The relevant sum-integrals are given in Appendix $\mathrm{A}$ Expanding the temperature-independent terms to order $\epsilon^{0}$, we find

$$
\begin{aligned}
& \mathcal{F}_{1 a}=-\frac{1}{4(4 \pi)^{2}}\left(\frac{\Lambda}{m_{\sigma}}\right)^{2 \epsilon}\left\{\left[\frac{1}{\epsilon}+\frac{3}{2}\right] m_{\sigma}^{4}+2 J_{0}\left(\beta m_{\sigma}\right) T^{4}\right\} \\
& \mathcal{F}_{1 b}=-\frac{(N-1)}{4(4 \pi)^{2}}\left(\frac{\Lambda}{m_{\pi}}\right)^{2 \epsilon}\left\{\left[\frac{1}{\epsilon}+\frac{3}{2}\right] m_{\pi}^{4}+2 J_{0}\left(\beta m_{\pi}\right) T^{4}\right\} \\
& \mathcal{F}_{1 c}=\frac{N_{c} N_{f}}{(4 \pi)^{2}}\left(\frac{\Lambda}{m_{q}}\right)^{2 \epsilon}\left\{\left[\frac{1}{\epsilon}+\frac{3}{2}\right] m_{q}^{4}-\left[K_{0}^{+}\left(\beta m_{q}, \beta \mu\right)+K_{0}^{-}\left(\beta m_{q}, \beta \mu\right)\right] T^{4}\right\},
\end{aligned}
$$

where $J_{0}(\beta m)$ and $K_{0}^{ \pm}\left(\beta m_{q}, \beta \mu\right)$ are defined in Appendix A. The divergent terms in $\mathcal{F}_{1 a-1 c}$ are thus

$$
\mathcal{F}_{1 a-1 c}^{\text {div }}=-\frac{1}{4(4 \pi)^{2} \epsilon}\left[m_{\sigma}^{4}+(N-1) m_{\pi}^{4} \quad-4 N_{c} N_{f} m_{q}^{4}\right] .
$$

In the minimal subtraction scheme the counterterm $\Delta \mathcal{F}_{1}$ cancels exactly the divergences in the free energy (28), which yields the one-loop counterterms

$$
\begin{aligned}
B_{1} & =\frac{N+2}{6(4 \pi)^{2}} \frac{\lambda}{\epsilon} \\
C_{1} & =\frac{1}{(4 \pi)^{2} \epsilon}\left[\frac{1}{6}(N+8) \lambda-24 N_{c} N_{f} \frac{g^{4}}{\lambda}\right], \\
D_{1} & =-\frac{N}{4(4 \pi)^{2}} \frac{1}{\epsilon}
\end{aligned}
$$

The final result for the renormalized one-loop free energy is obtained by adding Eqs. (25) - (27) and Eq. (21) using Eq. (29)-(31)

$$
\begin{aligned}
\mathcal{F}_{1}= & -\frac{1}{2} \chi v^{2}-\frac{1}{4(4 \pi)^{2}}\left\{\left[L_{\sigma}+\frac{3}{2}\right] m_{\sigma}^{4}+\left[L_{\pi}+\frac{3}{2}\right](N-1) m_{\pi}^{4}-\left[L_{q}+\frac{3}{2}\right] 4 N_{c} N_{f} m_{q}^{4}\right. \\
& \left.+2\left[J_{0}\left(\beta m_{\sigma}\right)+(N-1) J_{0}\left(\beta m_{\pi}\right)+2 N_{c} N_{f} K_{0}^{+}\left(\beta m_{q}, \beta \mu\right)+2 N_{c} N_{f} K_{0}^{-}\left(\beta m_{q}, \beta \mu\right)\right] T^{4}\right\}
\end{aligned}
$$


where we have defined

$$
L_{a} \equiv \log \frac{\Lambda^{2}}{m_{a}^{2}}, \quad a=\sigma, \pi, q .
$$

The full one-loop free energy $\mathcal{F}_{0+1}$ is then given by the sum of Eqs. (16) and (32). Using the renormalization group equation for the running coupling $\lambda$, one can show that $\mathcal{F}_{0+1}$ is renormalization group invariant.

\section{RESULTS AND DISCUSSION}

In this section we discuss the procedure for performing numerical calculations using OPT, and we present our results.

At one-loop order, the PMS condition on the free energy,

$$
\frac{d \mathcal{F}_{0+1}}{d \chi}=0
$$

simply has no solution.

Instead, we consider the gap equation for $v$, obtained by differentiating the free energy:

$$
\frac{d \mathcal{F}_{0+1}}{d v}=v\left(m_{\pi}^{2}+\Pi_{1}\right)-h=0 .
$$

Here, $\Pi_{1}$ is the one-loop pion self-energy at zero external momentum

$$
\Pi_{1}=\frac{1}{v} \frac{d \mathcal{F}_{1}}{d v} .
$$

A FAC condition on the self-energy is then $\Pi_{1}=0$, or ${ }^{2}$

$$
\chi=\frac{\lambda}{2} R\left(m_{\sigma}, T\right)+\frac{N-1}{3} \frac{\lambda}{2} R\left(m_{\pi}, T\right)+4 N_{c} N_{f} g^{2} S\left(m_{q}, T, \mu\right),
$$

where

$$
\begin{aligned}
R(m, T) & \equiv-\frac{m^{2}}{(4 \pi)^{2}}\left[L_{m}+1\right]+\frac{T^{2}}{(4 \pi)^{2}} J_{1}(\beta m), \\
S(m, T, \mu) & \equiv \frac{m^{2}}{(4 \pi)^{2}}\left[L_{m}+1\right]+\frac{T^{2}}{2(4 \pi)^{2}}\left[K_{1}^{+}(\beta m, \beta \mu)+K_{1}^{-}(\beta m, \beta \mu)\right] .
\end{aligned}
$$

This condition on $\chi$ has a straightforward physical interpretation - it means that Goldstone's theorem is fulfilled even at tree level:

$$
m_{\pi}^{2}=\frac{h}{v}
$$

This also means that we can write $m_{\sigma}^{2}=h / v+\lambda v^{2} / 3$, and thus all $\chi$-dependence is removed from the r.h.s. of Eq. 37 in the broken phase.

In order to perform numerical calculations, we need to find numerical values for the model parameters $m_{0}, \lambda, g$, and $\Lambda$ in terms of the vacuum $(T=\mu=0)$ values of $m_{\sigma}, m_{\pi}, m_{q}$, and $v$. Matching at tree level yields ${ }^{3}$

$$
\begin{aligned}
m_{0}^{2}+\chi & =-\frac{1}{2}\left(m_{\sigma}^{2}-3 m_{\pi}^{2}\right), \\
\lambda & =\frac{3}{v^{2}}\left(m_{\sigma}^{2}-m_{\pi}^{2}\right), \\
g & =\frac{m_{q}}{v} \\
h & =m_{\pi}^{2} v .
\end{aligned}
$$

\footnotetext{
${ }^{2}$ In some cases $\Pi_{1}=0$ has no solution. We then minimized $\left|\Pi_{1}\right|$ instead in accordance with the FAC condition.

${ }^{3}$ At tree level, it is the combination $m^{2}=m_{0}^{2}+\chi$ that is determined, cf. Eqs. (22)-23).
} 
At tree level, $\Lambda$ is undetermined. We choose the renormalization scale to be

$$
\Lambda^{2}=\frac{m_{\sigma}^{2}}{e} .
$$

Finally, we set $N=4, N_{f}=2$, and $N_{c}=3$.

\section{A. Contributions to $\mathcal{F}_{0+1}$}

At the one-loop level we distinguish between the following contributions to the free energy:

1. The tree-level contribution from the bosons,

$$
\mathcal{F}_{b}^{\text {tree }} \equiv \mathcal{F}_{0}=\frac{1}{2} m^{2} v^{2}+\frac{\lambda}{4 !} v^{4}-h v .
$$

2. The vacuum contribution from the bosons,

$$
\mathcal{F}_{b}^{\mathrm{vac}} \equiv-\frac{1}{4(4 \pi)^{2}}\left\{\left[L_{\sigma}+\frac{3}{2}\right] m_{\sigma}^{4}+\left[L_{\pi}+\frac{3}{2}\right](N-1) m_{\pi}^{4}\right\} .
$$

3. The thermal contribution from the bosons,

$$
\mathcal{F}_{b}^{T} \equiv-\frac{T^{4}}{2(4 \pi)^{2}}\left[J_{0}\left(\beta m_{\sigma}\right)+(N-1) J_{0}\left(\beta m_{\pi}\right)\right] .
$$

4. The vacuum contribution from the fermions,

$$
\mathcal{F}_{f}^{\mathrm{vac}} \equiv \frac{N_{c} N_{f} m_{q}^{4}}{(4 \pi)^{2}}\left[L_{q}+\frac{3}{2}\right] .
$$

5. The thermal contribution from the fermions,

$$
\mathcal{F}_{f}^{T} \equiv-\frac{N_{c} N_{f} T^{4}}{(4 \pi)^{2}}\left[K_{0}^{+}\left(\beta m_{q}, \beta \mu\right)+K_{0}^{-}\left(\beta m_{q}, \beta \mu\right)\right] .
$$

(There is also the OPT term, $-\frac{1}{2} \chi v^{2}$, which we omit for the purpose of the following discussion. It is taken into account in all numerical calculations.) We will now investigate the effect of these various contributions on the structure of the phase diagram.

\section{B. Chiral limit}

We start by considering the phase diagram in the chiral limit, $h=0$, where Lagrangian has an exact chiral symmetry and the pions are massless Nambu-Goldstone bosons. We then fix our model parameters using the vacuum values

$$
\begin{aligned}
m_{\sigma} & =600 \mathrm{MeV} \\
m_{\pi} & =0 \\
m_{q} & =300 \mathrm{MeV} \\
v & =f_{\pi}=93 \mathrm{MeV},
\end{aligned}
$$

and using Eqs. (40)-(44), we find that

$$
\begin{aligned}
m_{0}^{2}+\chi & =-(424.26 \mathrm{MeV})^{2}, \\
\lambda & =124.87, \\
g & =3.2258, \\
h & =0 \\
\Lambda & =363.92 \mathrm{MeV} .
\end{aligned}
$$


Starting with the simplest case, we may say that we are only interested in thermal effects, and thus ignore the vacuum contributions $\mathcal{F}_{b}^{\text {vac }}$ and $\mathcal{F}_{f}^{\text {vac }}$. Furthermore, it is common to use a mean-field approximation to the meson sector, in which we ignore the thermal fluctuations, $\mathcal{F}_{b}^{T}$, as well. Hence, all that remains of the free energy is the tree-level boson contribution and the thermal fluctuations of the quarks:

$$
\mathcal{F}=\mathcal{F}_{b}^{\text {tree }}+\mathcal{F}_{f}^{T}
$$

The phase diagram one obtains using this approximation is shown in Fig. 1 (left panel). We see that the phase transition is first order for all values of $\mu$, a fact which is explicitly demonstrated for $\mu=0$ in the right panel. The critical temperature is $T_{c}=140 \mathrm{MeV}$, which is reasonably close to the critical temperature of QCD. The critical chemical potential for $T=0$ is $\mu_{c}=296 \mathrm{MeV}$.
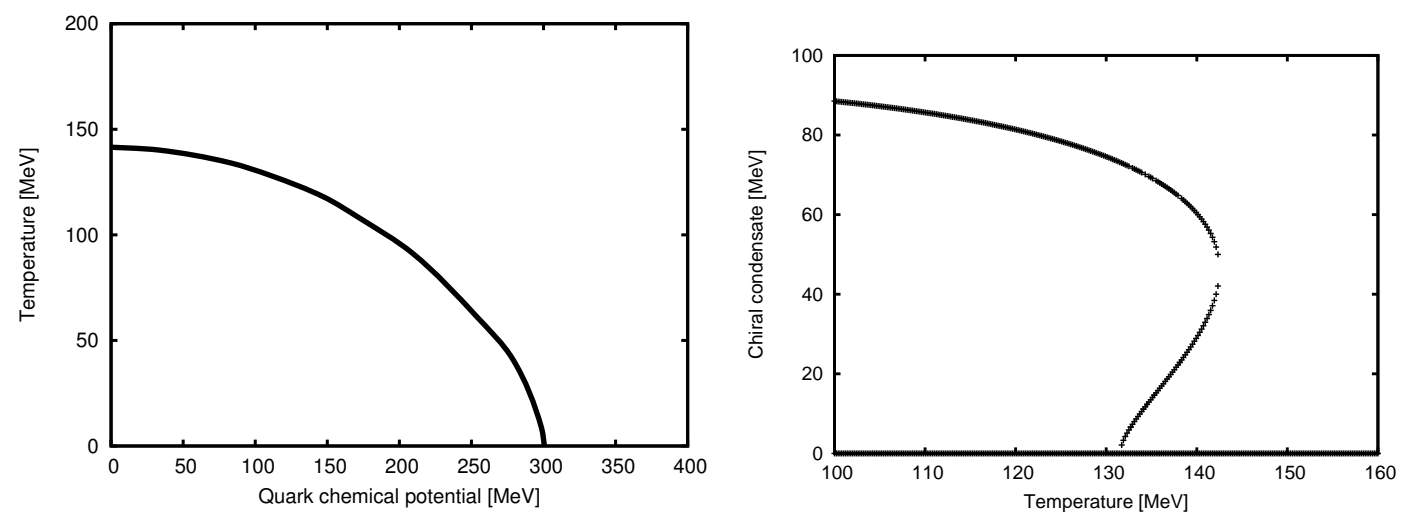

FIG. 1: At one loop, including only the thermal contribution of the fermions and the tree-level contribution of the bosons: (left panel) the phase diagram in the $\mu-T$ plane, where the solid line represents a first-order phase transition, and (right panel) the solutions of the gap equation plotted as a function of $T$ at $\mu=0$.

Recently, Skokov et al [26] showed analytically for $\mu=0$ that if one includes the fermion vacuum term, i.e.

$$
\mathcal{F}=\mathcal{F}_{b}^{\text {tree }}+\mathcal{F}_{f}^{\mathrm{vac}}+\mathcal{F}_{f}^{T}
$$

the order of the phase transition changes - it becomes a second-order phase transition. For $T=0$ and finite $\mu$, this can be shown analytically as well. This is displayed in the right panel of Fig. 2. Furthermore, as the left panel shows, the phase transition is of second order for all values of $\mu$. The critical temperature at $\mu=0$ is $T_{c}=190 \mathrm{MeV}$, a significant increase as compared to the case where the fermion vacuum fluctuations were ignored. Similarly, there is a large increase in the critical chemical potential for $T=0$ to $\mu_{c}=345 \mathrm{MeV}$.
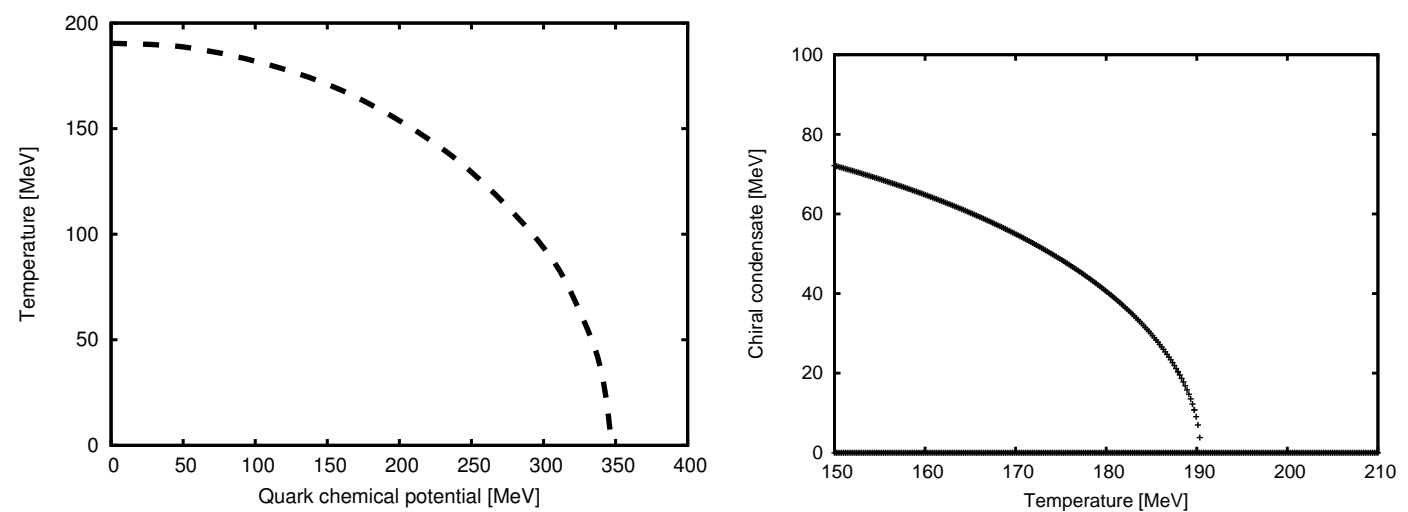

FIG. 2: At one loop, including both the vacuum contribution and the thermal contribution of the fermions, but only the tree-level contribution of the bosons: (left panel) the phase diagram in the $\mu-T$ plane, where the dashed line represents a second-order phase transition, and (right panel) the solutions of the gap equation plotted as a function of $T$ at $\mu=0$. 
Next, let us omit the fermionic vacuum fluctuations again, and instead study the effects of including the thermal fluctuations of the mesons:

$$
\mathcal{F}=\mathcal{F}_{b}^{\text {tree }}+\mathcal{F}_{b}^{T}+\mathcal{F}_{f}^{T}
$$

This situation was investigated by Bowman and Kapusta in Ref. 25]. In the chiral limit, they found that there is a first-order phase transition extending all the way from the $\mu$ axis to the temperature axis, with a critical temperature of $T_{c} \sim 140 \mathrm{MeV}$ at $\mu=0$. We show this in Fig. 3, where we also see that we get a lower critical temperature of about $110 \mathrm{MeV}$. The critical chemical potential is for $T=0$ is $\mu_{c}=357 \mathrm{MeV}$.
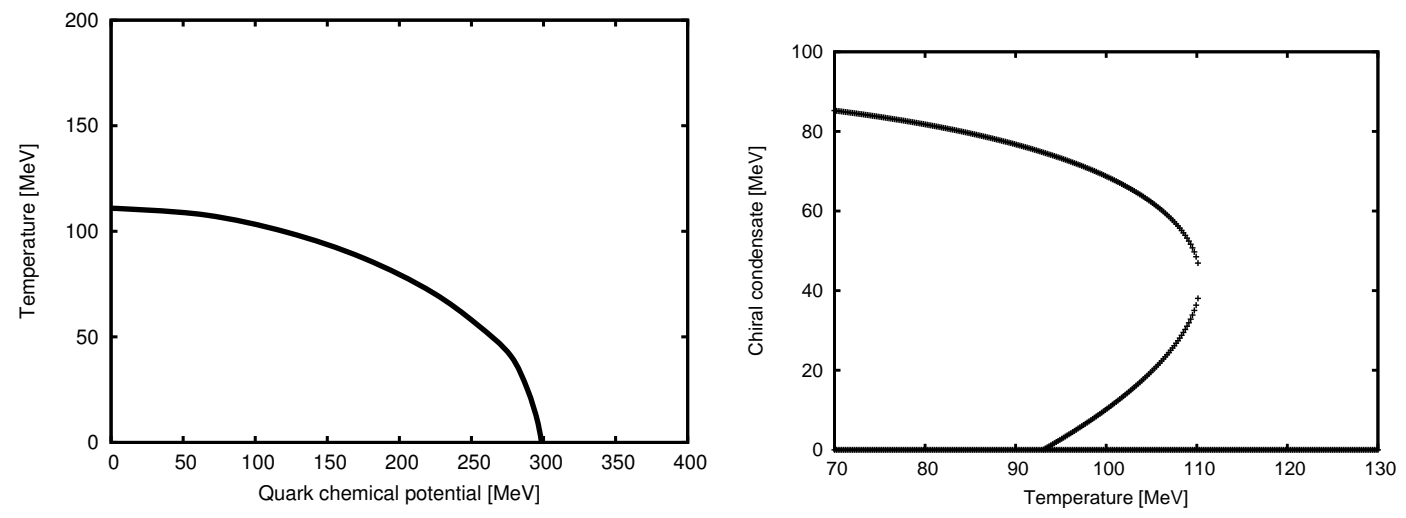

FIG. 3: At one loop, including the thermal contributions from both fermions and bosons, but not vacuum contributions: (left panel) the phase diagram in the $\mu-T$ plane, where the solid line represents a first-order phase transition, and (right panel) the solutions of the gap equation plotted as a function of $T$ at $\mu=0$.

We have now seen that by including $\mathcal{F}_{f}^{\text {vac }}$ the first-order phase transition turns into the second-order transition one would expect at high temperatures, and that by taking $\mathcal{F}_{b}^{T}$ into account we pushed the critical temperature down to the vicinity of $T_{c}^{\mathrm{QCD}}$. It is therefore tempting to guess that by including all contributions,

$$
\mathcal{F}=\mathcal{F}_{b}^{\text {tree }}+\mathcal{F}_{b}^{\text {vac }}+\mathcal{F}_{b}^{T}+\mathcal{F}_{f}^{\text {vac }}+\mathcal{F}_{f}^{T}
$$

we will get a second-order phase transition near $T=150 \mathrm{MeV}$. Unfortunately, this turns out not to be the case. As Fig. 4 shows, the critical temperature is $148 \mathrm{MeV}$, but the phase transition is still first order - in fact it is even more strongly so. Part of the reason for this is the fact that the vacuum energy of the mesons has opposite sign from the vacuum energy of the quarks and so the contributions cancel to some extent. The critical chemical for potential for $T=0$ is $\mu_{c}=357 \mathrm{MeV}$.
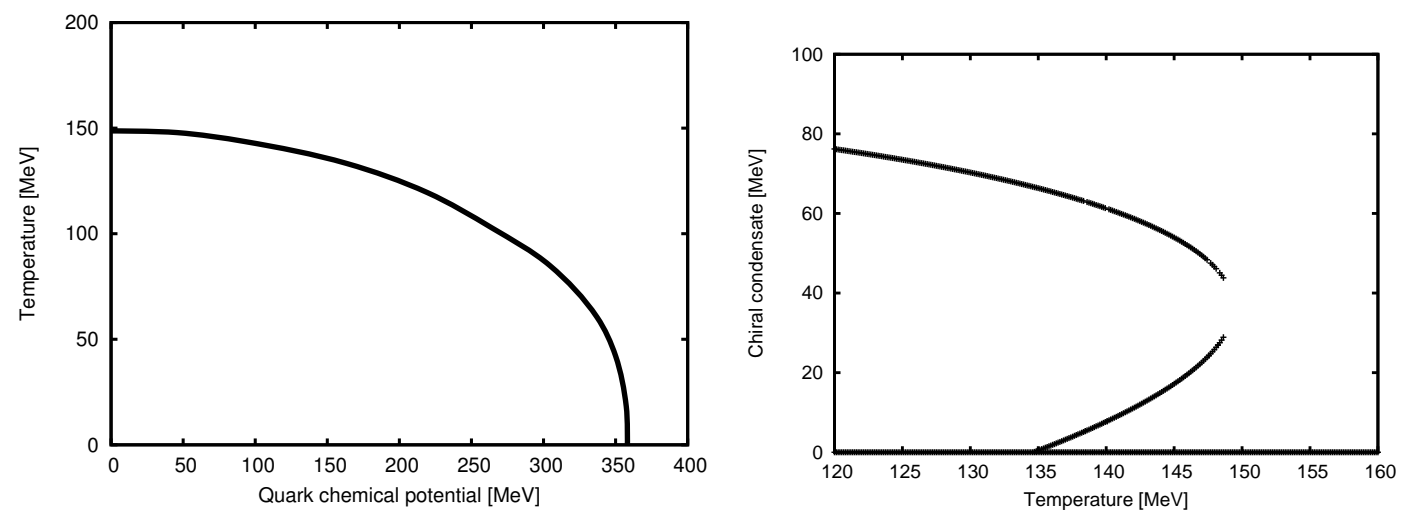

FIG. 4: At one loop, including all contributions from both fermions and bosons: (left panel) the phase diagram in the $\mu-T$ plane, where the solid line represents a first-order phase transition, and (right panel) the solutions of the gap equation plotted as a function of $T$ at $\mu=0$. 


\section{Physical point}

In the chiral limit, $v$ is exactly zero in the chirally restored phase. If we let $h>0$, chiral symmetry is explicitly broken in the Lagrangian. Then, the chiral condensate never vanishes completely, but approaches zero asymptotically at high temperature and/or large baryon chemical potential. Furthermore, the pions acquire mass and become pseudo Nambu-Goldstone bosons.

At the physical point, we use the vacuum values

$$
\begin{aligned}
m_{\sigma} & =600 \mathrm{MeV}, \\
m_{\pi} & =139 \mathrm{MeV}, \\
m_{q} & =300 \mathrm{MeV}, \\
v & =f_{\pi}=93 \mathrm{MeV},
\end{aligned}
$$

and using Eqs. (40)- (44) we find the parameters

$$
\begin{aligned}
m_{0}^{2}+\chi & =-(388.611 \mathrm{MeV})^{2} \\
\lambda & =118.17 \\
g & =3.2258 \\
h & =(121.573 \mathrm{MeV})^{3} \\
\Lambda & =363.92 \mathrm{MeV}
\end{aligned}
$$

Let us look at what happens to the phase transition in the case when only thermal contrubtions to the free energy are taken into account.

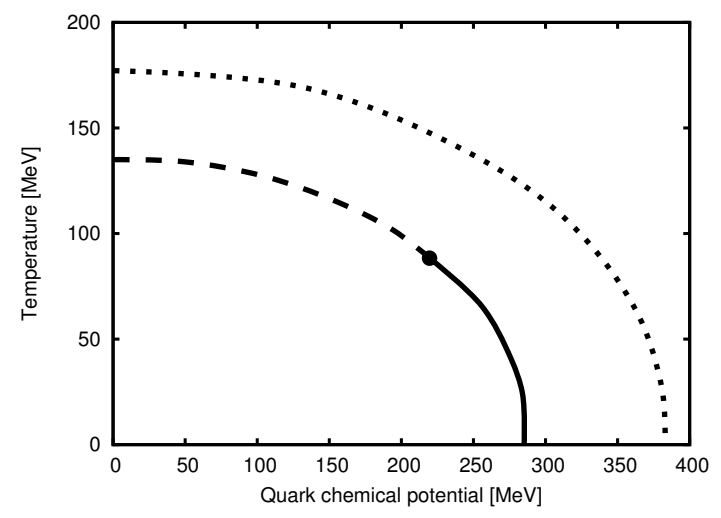

FIG. 5: At one loop, including all contributions from both fermions and bosons (upper curve) and thermal contributions only (lower curve).

This is shown in Fig 5 (lower curve). The line of first-order transition that starts at $\mu_{c}=287 \mathrm{MeV}$ and $T=0$ ends at a critical point at $\mu=220 \mathrm{MeV}$ and $T=90 \mathrm{MeV}$. In comparison, Scavenius $[13]$ et al. obtain $\mu=207 \mathrm{MeV}$ and $T=99 \mathrm{MeV}$ for the position of the critical endpoint, while Bowman and Kapusta [25] obtain the values $\mu=283 \mathrm{MeV}$ and $T=75 \mathrm{MeV}$.

If we include the bosonic as well as the fermionic vacuum contributions, i.e. we include all contributions in the model, the critical endpoint disappears. The transition is a crossover in the whole $\mu-T$ plane. This is shown in Fig [5] as well for comparison (upper curve). Only for lower values of $m_{\pi}$ will the critical endpoint appear.

\section{SUMMARY AND OUTLOOK}

In the present paper, we have applied optimized perturbation theory to the quark-meson model to study the chiral transition in the $\mu-T$ plane. The properties of the phase transition - the order and the critical temperature - depend crucially on the the approximations made. The one-loop calculation in the chiral limit yields either a first-order or a 
second-order transition in the whole $\mu-T$ plane depending on whether one includes the fermion vacuum fluctuation term or not. This is in contrast to most NJL-type model calculations [4] and functional renormalization group calculations that predict a curve of first-order transitions starting at $T=0$ ending at at critical point [21, 22, 36]. Taking a model seriously means including the effects of all its degrees of freedom. It therefore questionable to throw out some terms unless one can show that they are not important. For example, the effective potential is receiving contributions from vacuum fluctuations from all energies scales up to the ultraviolet cutoff $\Lambda$ of the effective theory. This is the region of validity of the low-energy effective theory and it is therefore not obvious that they can be omitted. In view of this, it is perhaps somewhat surprising that the critical endpoint disappears all together at the physical point and we are left with a crossover in the whole $\mu-T$ plane.

We close with a few remarks concerning future work. Optimized perturbation theory was used at the two-loop level in $\phi^{4}$-theory to study the phase transition and it was shown that it correctly reproduces the second-order nature at zero baryon chemical potential [10]. It would therefore be of interest to extend the two-loop calculations to the quark-meson model at finite $\mu$ along the lines of Ref. [29]. Secondly, one could apply OPT to the case $N_{f}=3$, where we know the transition in the chiral limit is first order. Dialing the strange quark mass from $m_{s}=\infty$ to $m_{s}=0$ interpolate between the chiral limit for $N_{f}=2$ and $N_{f}=3$. Somewhere along the way, the transition then changes

from second order to first order and it would be interesting to find the critical value for the strange quark mass, $m_{s}^{*}$ where this happens.

\section{Appendix A: One-loop sum-integrals}

We need the following bosonic sum-integrals

$$
\begin{gathered}
\mathcal{F}_{P} \log \left(P^{2}+m^{2}\right)=\frac{1}{(4 \pi)^{2}}\left(\frac{\Lambda}{m}\right)^{2 \epsilon}\left[-\frac{e^{\gamma \epsilon} \Gamma(1+\epsilon)}{\epsilon(1-\epsilon)(2-\epsilon)} m^{4}-J_{0}(\beta m) T^{4}\right], \\
\mathcal{F}_{P} \frac{1}{P^{2}+m^{2}}=\frac{1}{(4 \pi)^{2}}\left(\frac{\Lambda}{m}\right)^{2 \epsilon}\left[-\frac{e^{\gamma \epsilon} \Gamma(1+\epsilon)}{\epsilon(1-\epsilon)} m^{2}+J_{1}(\beta m) T^{2}\right] .
\end{gathered}
$$

The temperature-dependent term is expressed as integrals over the Bose-Einstein distribution function:

$$
J_{n}(x)=\frac{4 e^{\gamma \epsilon} \Gamma\left(\frac{1}{2}\right)}{\Gamma\left(\frac{5}{2}-n-\varepsilon\right)} x^{4-2 n} \int_{0}^{\infty} d t \frac{t^{4-2 n-2 \epsilon}}{\sqrt{t^{2}+1}} \frac{1}{e^{x \sqrt{t^{2}+1}}-1} .
$$

These integrals satisfy the recursion relation

$$
\frac{d J_{n}}{d x}=2 \epsilon \frac{J_{n}(x)}{x}-2 x J_{n+1}(x) .
$$

We need the following fermionic sum-integrals:

$$
\mathcal{F}_{\{P\}} \operatorname{tr} \log \left(i \gamma_{\mu} P_{\mu}+m-\mu \gamma_{4}\right)=\frac{1}{(4 \pi)^{2}}\left(\frac{\Lambda}{m}\right)^{2 \epsilon}\left[-2 \frac{e^{\gamma \epsilon} \Gamma(1+\epsilon)}{\epsilon(1-\epsilon)(2-\epsilon)} m^{4}+\left[K_{0}^{+}(\beta m, \beta \mu)+K_{0}^{-}(\beta m, \beta \mu)\right] T^{4}\right] .
$$

Here, the temperature-dependent term is expressed as integrals over the Fermi-Dirac distribution function:

$$
K_{n}^{ \pm}(x, y)=\frac{4 e^{\gamma \epsilon} \Gamma\left(\frac{1}{2}\right)}{\Gamma\left(\frac{5}{2}-n-\varepsilon\right)} x^{4-2 n} \int_{0}^{\infty} d t \frac{t^{4-2 n-2 \epsilon}}{\sqrt{t^{2}+1}} \frac{1}{e^{x \sqrt{t^{2}+1} \pm y}+1} .
$$

The functions $K_{n}^{ \pm}(x, y)$ satisfy the same recursion relation as $J_{n}(x)$,

[1] R. D. Pisarski and F. Wilczek, Phys. Rev. D 29 (1984) 338.

[2] M. A. Stephanov, PoS LAT2006, (2006) 024. 
[3] S. D. H. Hsu and M. Schwetz, Phys. Lett. B 432, 203 (1998).

[4] M. Buballa, Phys. Rept. 407, (2005), 205.

[5] A. Bazavov et al., Phys. Rev. D 80, (2009), 014504.

[6] S. Borsanyi et al., JHEP 1011, (2010), 077

[7] O. Philipsen. arXiv:1009.4089 (2010).

[8] S. Chiku and T. Hatsuda. Phys. Rev. D58 (1998), 076001.

[9] J. T. Lenaghan and D. H. Rischke, J. Phys. G 26, (2000), 431; J. T. Lenaghan, D. H. Rischke, and J. Schaffner-Bielich, Phys. Rev. D 62, (2000), 085008.

[10] S. Chiku. Prog. Theor. Phys. 104, (2000), 1129.

[11] T. Herpay and Zs. Szep. Phys. Rev. D 74, (2006), 025008.

[12] R. L. S. Farias, G. Krein, and R. O. Ramos. Phys. Rev. D 78, (2008), 065046.

[13] O. Scavenius, A. Mocsy, I. N. Mishustin, and D. H. Rischke, Phys. Rev. C 64 (2001), 045202.

[14] J.-L Kneur, M. B. Pinto, and R. O. Ramos, Phys. Rev. C 81, (2010), 065205.

[15] P. Costa, M.C. Ruivo, and C.A. de Sousa, Phys. Rev. D 77, (2008), 096001.

[16] T. Kahara and K. Tuominen, Phys. Rev. D 78, (2008), 034015; ibid D 82, (2010), 114026.

[17] D. Nickel, Phys.Rev. D 80, (2009), 074025.

[18] P. Costa, M. C. Ruivo, C. A. de Sousa, and H. Hansen, Symmetry 2, (2010), 1338.

[19] A. Jakovac, A. Patkos, Z. Szep, and P. Szepfalusy, Phys. Lett. B 582, (2004), 179.

[20] A. Mocsy, I. N. Mishustin, and P. J. Ellis, Phys. Rev. C 70, (2004), 015204.

[21] B.-J. Schaefer and J. Wambach, Nucl. Phys. A 757, (2005), 479.

[22] B.-J. Schaefer and J. Wambach, Phys. Rev. D 75, (2007), 085015.

[23] P. Kovacs and Zs. Szep. Phys. Rev. D 75, (2007), 025015.

[24] B.-J. Schaefer and M. Wagner, Phys. Rev. D 79, (2009), 014018.

[25] E. S. Bowman and J. I. Kapusta, Phys. Rev. C 79, (2008), 015202.

[26] V. Skokov, B. Friman, E. Nakano, K. Redlich, and B.-J. Schaefer, Phys. Rev. D 82 (2010), 034029.

[27] A. Jakovac and Zs. Szep, Phys. Rev. D 82, (2010), 125038.

[28] L. Ferroni, V. Koch, and M. B. Pinto, Phys. Rev. C 82, (2010) 055205.

[29] E. S. Fraga, L. F. Palhares, and M. B. Pinto. Phys. Rev. D79 ,(2009), 065026.

[30] K. Fukushima, Phys. Lett. B 591, (2004), 277.

[31] B.-J. Schaefer, J. M. Pawlowski, and J. Wambach. Phys. Rev. D 76, (2007), 074023.

[32] K. Kashiwa, H. Kouno, and M. Matsuzaki, and M. Yahiro Phys. Lett. B 662, (2008), 26.

[33] H. Mao, J. Jin, and M. Huang, J. Phys. G 37 (2010), 035001.

[34] U. S. Gupta and V. K.Tiwari, Phys. Rev. D 81, (2010), 054019.

[35] G. Marko and Zs. Szep, Phys. Rev. D 82, (2010), 065021.

[36] T. K. Herbst, J. M. Pawlowski, and B.-J. Schaefer. Phys. Lett. B 696, 58 (2011).

[37] A. E. Radzhabov, D. Blaschke, M. Buballa, and M. K. Volkov, e-Print: arXiv:1012.0664 [hep-ph].

[38] M. G. Alford, A. Schmitt, and K. Rajagopal, Rev. Mod. Phys. 80, (2008), 1455.

[39] A. J. Mizher and E. S. Fraga. Nucl. Phys. A 831, (2009) 91.

[40] V. I. Yukalov, Teor. Mat. Fiz. 26 (1976), 403.

[41] P. M. Stevenson, Phys. Rev. D 23 (1981), 2916.

[42] A. Duncan and M. Moshe, Phys. Lett. B 215 (1988), 352.

[43] A. Duncan and H. F. Jones, Phys. Rev. D 47 (1993), 2560.

[44] H. Kleinert, Path Integrals in Quantum Mechanics, Statistics, and Polymer Physics, 2nd edition, World Scientific Publishing Co., Singapore, (1995).

[45] A. N. Sisakian, I. L. Solovtsov and O. Shevchenko, Int. J. Mod. Phys. A 9 (1994), 1929.

[46] W. Janke and H. Kleinert, Phys. Rev. Lett. 75, (1995), 2787.

[47] F. Karsch, A. Patkos and P. Petreczky, Phys. Lett. B 401, (1997), 69. Phys. Lett. B401, (1997), 69.

[48] J. O. Andersen, E. Braaten, and M. Strickland, Phys. Rev. D 63, (2001), 105008. 
